Quim. Nova, Vol. 32, No. 8, 2114-2121, 2009

\title{
ESTUDOS DE QSAR 3D PARA UM CONJUNTO DE INIBIDORES DE BUTIRILCOLINESTERASE HUMANA
}

\author{
Humberto F. Freitas, Odailson S. Paz e Marcelo S. Castilho* \\ Departamento de Medicamentos, Faculdade de Farmácia, Universidade Federal da Bahia, Campus Universitário de Ondina, \\ 40170-115 Salvador - BA, Brasil
}

Recebido em 9/12/08; aceito em 14/4/09; publicado na web em 22/9/09

\begin{abstract}
QSAR 3D STUDIES OF A SERIES OF HUMAN BUTYRYLCHOLINESTERASE INHIBITORS. Alzheimer's disease (AD) is considered the main cause of cognitive decline in adults. The available therapies for AD treatment seek to maintain the activity of cholinergic system through the inhibition of the enzyme acetylcholinesterase. However, butyrylcholinesterase (BuChE) can be considered an alternative target for AD treatment. Aiming at developing new BuChE inhibitors, robust QSAR 3D models with high predictive power were developed. The best model presents a good fit $\left(r^{2}=0.82, q^{2}=0.76\right.$, with two PCs $)$ and high predictive power $\left(r_{\text {predict }}^{2}=0.88\right)$. Analysis of regression vector shows that steric properties have considerable importance to the inhibition of the BuChE.
\end{abstract}

Keywords: butyrylcholinesterase; Alzheimer; QSAR 3D.

\section{INTRODUÇÃO}

A doença ou mal de Alzheimer (DA), descrita primeiramente por Alois Alzheimer (1864-1915) ${ }^{1}$ acomete cerca de 37 milhões de pessoas em todo o mundo, ${ }^{2}$ sendo considerada a principal causa de declínio cognitivo em adultos. ${ }^{3}$ De fato, 50 a $70 \%$ dos casos de demência entre idosos acima de 65 anos está relacionada à DA e aproximadamente $5 \%$ desta população será afetada pelo mal de Alzheimer. ${ }^{4}$ De acordo com a OMS, a prevalência da doença dobra a cada 5 anos, passando de 3\% aos 70 anos para 20-30\% aos 85 anos. ${ }^{3}$ Dessa forma, esperase que o número de casos aumente para 114 milhões até $2050 .^{5}$ De fato, em função do número crescente de pacientes com DA, os custos diretos e indiretos associados ao tratamento dessa doença são de, no mínimo, 100 bilhões de dólares, nos EUA. ${ }^{2}$

O mal de Alzheimer caracteriza-se por diversos eventos metabólicos que culminam no aparecimento dos sinais característicos da doença. Entre esses eventos estão a deposição de placas de proteínas insolúveis $\beta$-Amiloides ( $\mathrm{Ab}$ ), como resultado do metabolismo anormal da proteína precursora de $\beta$-Amiloide (PPA) e formação de emaranhados neurofibrilares, em função do colapso do citoesqueleto neuronal, decorrente da hiperfosforilação da proteína Tau, em regiões do lobo temporal e neocórtex. ${ }^{6}$ Soma-se a isso, o processo de disfunção simpática e neuroquímica relacionada à redução da atividade colinérgica central. ${ }^{7}$ Como consequência desses eventos neuropatológicos, há perda de memória, grandes variações de humor, depressão associada a distúrbios de linguagem, perda de poder de julgamento etc.

Atualmente, as principais estratégias terapêuticas para o tratamento de DA baseiam-se na hipótese colinérgica ou na hipótese amiloide. De acordo com a hipótese colinérgica, processos neurodegenerativos levam à destruição de neurônios colinérgicos da região frontal do cérebro resultando em déficit da transmissão colinérgica central, ${ }^{4}$ provocando declínio cognitivo e perda da capacidade de execução das tarefas diárias, redução da atenção e da memória. ${ }^{8-10}$ Segundo a hipótese da cascata amiloide, os níveis elevados de proteínas insolúveis $\beta$-Amiloides (Ab) são responsáveis pela formação de placas amiloides e emaranhados neurofibrilares que estão relacionados com morte celular, processos inflamatórios, demência e DA. ${ }^{11}$ Embora o desenvolvimento de fármacos que bloqueiem ou revertam a formação de proteínas insolúveis $\beta$ Amiloides seja uma área de grande interesse, ${ }^{12,13}$ as terapias disponíveis

*e-mail: castilho@ufba.br atualmente para o tratamento de DA visam manter e facilitar a atividade dos sistemas colinérgicos sobreviventes. ${ }^{14}$ Nesse sentido, a principal estratégia terapêutica disponível se baseia na inibição das colinesterases (ChEIs), uma vez que a modulação dos níveis de ACh é feita por duas enzimas, a acetilcolinesterase (AChE - EC 3.1.1.7) e butirilcolinesterase (BuChE - EC 3.1.1.8). ${ }^{15}$ Ainda que essas enzimas possuam identidade sequencial acima de $50 \%$ e um sítio catalítico acessível através de um canal com aproximadamente $20 \AA$ de profundidade, o volume interno o sítio ativo da $\mathrm{AChE}$ é relativamente pequeno $\left(302 \mathrm{~A}^{3}\right)$, ao passo que $\mathrm{BuChE}$ possui um sítio ativo de volume consideravelmente maior (502 $\left.\mathrm{A}^{3}\right) .{ }^{16}$ Essa diferença é consequência dos diferentes aminoácidos presentes em cada um dos sítios e está diretamente ligada à acomodação e seletividade dos diferentes inibidores por AChE ou BuChE. ${ }^{16}$

Atualmente há quatro fármacos no mercado, doneprezil (Aricept; Eisai/Pfizer), rivastigmina (Exelon; Novartis), galantamina (Razadyne; Johnson \& Johnson) e tacrina (Cognex; First Horizon Pharmaceuticals) ${ }^{17}$ (Figura 1), que são inibidores de acetilcolinesterase. No entanto, em decorrência do desenvolvimento da DA há um declínio dos níveis de expressão de $\mathrm{AChE}$, associada a uma marcante elevação dos níveis de BuChE ${ }^{14,18}$ Esses resultados sugerem que, em pacientes com DA, os níveis de $\mathrm{ACh}$ sejam controlados principalmente por BuChE. ${ }^{19}$ Nesse contexto, fármacos que inibam tanto acetilcolinesterase como butirilcolinesterase podem ter efeitos benéficos no tratamento sintomático de DA. ${ }^{20}$

$\mathrm{Na}$ tentativa de melhor caracterizar inibidores de butirilcolineterase humana, Castilho e colaboradores desenvolveram modelos de QSAR 2D, utilizando a técnica de holograma QSAR. ${ }^{21}$ Embora os resultados obtidos nesse trabalho sejam úteis no planejamento de novos inibidores de BuChE, os mapas de contribuição do holograma QSAR, previamente descritos, não permitem uma atribuição clara das razões para contribuição favorável ou desfavorável dos fragmentos moleculares. Visando aprofundar o estudo sobre esse sistema, esse artigo descreve o desenvolvimento e validação de modelos de QSAR $3 \mathrm{D}$ robustos e com alto poder preditivo para inibidores de butirilcolinesterase humana utilizando descritores topográficos.

\section{PARTE EXPERIMENTAL}

\section{Conjunto de inibidores}

Foi utilizado um conjunto de 53 inibidores de butirilcolinesterase (BuChE), derivados de tacrina e huprina, previamente descritos na 
literatura $^{22-28}$ (Tabela 1), cuja potência varia entre 1,48 e $80.000 \mathrm{nM}$. Os valores de $\mathrm{IC}_{50}$ foram convertidos em $\mathrm{pIC}_{50}\left(-\log \mathrm{IC}_{50}\right)$ e usados como variável dependente na análise de QSAR. Estas moléculas tiveram suas estruturas químicas desenhadas em formato $2 \mathrm{D}$ e depois convertidas para formato 3D, utilizando a plataforma Sybyl 7.2. A esteroquímica (7R11R, 7S11S e 7R11S) foi imposta manualmente a todos os compostos e nenhuma restrição à flexibilidade das ligações químicas foi imposta. A seguir, todas as estruturas foram otimizadas utilizando o método semiempírico AM1 (Palavras-chave: 1SCF XYZ ESP NOINTER SCALE $=1.4 \mathrm{NSURF}=2 \mathrm{SCINCR}=0.4)$. Os inibidores foram divididos em grupo teste (1-44) e grupo treino (45-53) para fins de validação externa. Os critérios para divisão do conjunto de dados foram: diversidade estrutural observada na análise hierárquica de cluster (HCA) (Figura 2) e variação de potência dessas moléculas. Para a geração do HCA, as variáveis foram autoescalonadas e agrupadas através do método de ligação ward, utilizando distâncias Euclidanas, como disponível no programa MINITAB 14.

\section{Alinhamento molecular}

A criação de modelos de QSAR 3D é profundamente dependente das conformações utilizadas. ${ }^{29}$ Por essa razão, estratégias baseadas na estrutura do alvo macromolecular e com base na estrutura do ligante foram utilizadas. A criação dos modelos com base na estrutura do alvo molecular foi feita a partir da estrutura cristalográfica de butirilcolinesterase em complexo com ácido butanoico (código PDB 2J4C). $\mathrm{O}$ espaço de busca foi delimitado a partir do centro do sítio ativo de butirilcolinesterase (coordenadas $\mathrm{X}=136,45 ; \mathrm{Y}=122,34 ; \mathrm{Z}=40,15$ ) utilizando-se um raio de $10 \AA$. Durante o acoplamento molecular, os inibidores foram considerados como flexíveis, exceto pelos seus anéis, que o programa GOLD 3.11 considera como rígidos, enquanto os resíduos da proteína foram considerados como fixos. Uma molécula de água (HOH531), com mobilidade parcial, foi considerada durante a criação dos modelos de interação inibidor-enzima. Os modelos de interação foram gerados através de algoritmo genético utilizando a opção standard, disponível no programa GOLD 3.11. Resumidamente: 250 indivíduos (conformações), de cada um dos 53 inibidores, evoluíram por 250.000 gerações, sofrendo taxa de mutação e crossover de $95 \%$. O indivíduo melhor adaptado (conformação) de cada inibidor é aquele que apresenta maior complementaridade estérica e eletrônica com o sítio ativo de butirilcolinesterase. A adaptação dos indivíduos foi avaliada através da função Goldscore. A fim de selecionar a melhor solução, as três conformações de maior pontuação, para cada inibidor, foram reavaliadas com auxílio do programa Drugscore ${ }^{30-33}$ disponível em http://pc1664.pharmazie.uni-marburg. de/drugscore/, e analisadas visualmente, com auxílio do programa PYMOL. Somente a conformação de cada inibidor que apresentou melhor resultado nesta etapa de avaliação visual (CONF-GOLD) foi utilizada nos estudos subsequentes.

As conformações com base no ligante foram obtidas a partir da sobreposição de cada uma das 53 moléculas do conjunto de inibidores sobre a molécula de huprina. Essa estratégia leva em consideração que todos os inibidores devem ocupar um volume similar e realizar interações semelhantes no sítio ativo da macromolécula. A fim de realizar esse procedimento utilizaram-se os programas OMEGA e ROCS empregando parâmetros padrão, ${ }^{34}$ exceto nos casos descritos a seguir. Resumidamente; o programa OMEGA foi utilizado para gerar um banco de dados contendo multiconfôrmeros dos 53 inibidores da BuChE. Os confôrmeros são gerados a partir da torsão das ligações entres os átomos levando em consideração o campo de força molecular MMFF94 e uma janela de energia de $15 \mathrm{Kcal} / \mathrm{mol}$. No passo seguinte, utilizou-se o programa ROCS para sobrepor todos os confôrmeros de cada molécula sobre a estrutura de huprina A, obtida a partir da estrutura cristalográfica 1VOT. As sobreposições foram feitas de forma a maximizar a sobreposição dos volumes moleculares levando em consideração a sobreposição de átomos com características químicas semelhantes (CONF-COMBO), como disponível através da palavra chave -rankby combo-score. Propriedades eletrônicas não foram consideradas para a sobreposição.

\section{Cálculo e seleção de descritores}

O programa DRAGON 5.4 foi utilizado para calcular descritores 3D (N=678) (WHIM, GETAWAY e MORSE) para os inibidores nas conformações CONF-GOLD e CONF-COMBO. Descritores com valor constante, baixa variância $(<0,01)$ ou com alta correlação entre si $(>0,97)$ foram excluídos por não contribuírem informações relevantes para o desenvolvimento de modelos de QSAR 3D. Esse procedimento resultou na seleção de 277 descritores para CONFGOLD e 282 para CONF-COMBO. A seguir utilizou-se o programa BUILDQSAR para geração de modelos de regressão linear múltipla (RLM), utilizando no máximo 4 descritores, através de busca sistemática. Somente modelos com $r^{2}>0,78$ foram considerados. As variáveis independentes (descritores) presentes nos dez melhores modelos (17 descritores para CONF-GOLD e 29 descritores para CONF-COMBO) foram agrupadas, autoescalonadas e utilizadas para análise exploratória dos dados utilizando análise de componentes principais (PCA), agrupamento hierárquico de grupos (HCA) e geração de modelos de regressão por mínimos quadrados parciais (PLS).

\section{Desenvolvimento e validação dos modelos de QSAR 3D}

A seleção do número ótimo de componentes principais (PCs) foi feita pelo método de validação cruzada Leave one out (LOO) $\left(q^{2}\right)$, enquanto que o poder preditivo dos modelos foi avaliado através de validação externa, utilizando-se as moléculas do grupo teste, não utilizadas durante o desenvolvimento dos modelos de QSAR 3D. Durante o desenvolvimento dos modelos de QSAR, a importância de cada descritor para PLS foi avaliada através da análise do vetor de regressão de cada descritor em relação ao modelo.

\section{RESULTADOS E DISCUSSÃO}

Um conjunto de 53 inibidores de BuChE, derivados de tacrina e huprina (Figura 1), foram selecionados a partir da literatura. ${ }^{22-28} \mathrm{~A}$ estrutura química e potência, que varia entre 1,48 a $88.000 \mathrm{nM}$ (diferença de 60.000 vezes), do conjunto completo de inibidores estão representadas na Tabela 1 . O valores de $\mathrm{IC}_{50}$ foram convertidos para $\mathrm{pIC}_{50}\left(-\log \left[\mathrm{IC}_{50}\right]\right)$ e usados como variáveis dependentes durante o desenvolvimento dos modelos de QSAR 3D. A primeira etapa para o desenvolvimento de modelos de QSAR 3D é a obtenção de estruturas 3D que representem a conformação bioativa das moléculas em estudo. Existem várias técnicas que podem ser utilizadas para tal finalidade, ${ }^{35}$

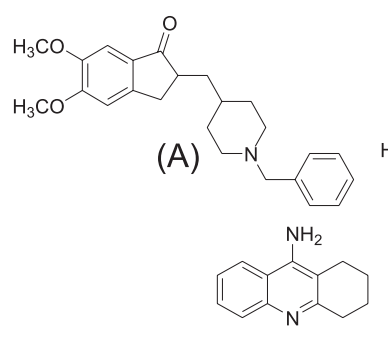

(D)
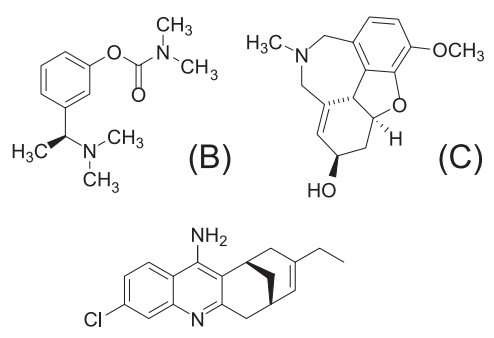

(E)
Figura 1. Fármacos utilizados no tratamento do Mal de Alzheimer. Doneprezil $(A)$, rivastigmina $(B)$, galantamina $(C)$, tacrina $(D)$ e huprina $(E)$ 
Tabela 1. Moléculas do grupo treino e grupo teste

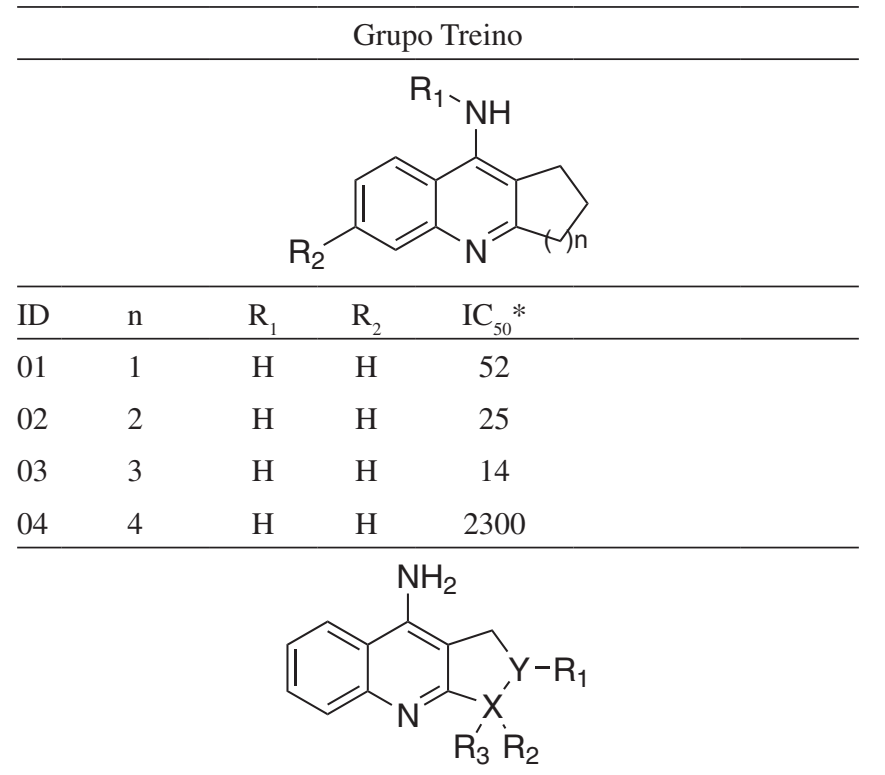

\begin{tabular}{ccccccc}
\hline $\mathrm{ID}$ & $\mathrm{Y}$ & $\mathrm{X}$ & $\mathrm{R}_{1}$ & $\mathrm{R}_{2}$ & $\mathrm{R}_{3}$ & $\mathrm{IC}_{50}{ }^{*}$ \\
\hline 05 & $\mathrm{CH}$ & $\mathrm{C}$ & $\mathrm{CH}_{3}$ & $\mathrm{CH}_{3}$ & $\mathrm{OH}$ & 12200 \\
06 & $\mathrm{CH}$ & $\mathrm{C}$ & $\mathrm{CH}_{3}$ & n-But & $\mathrm{OH}$ & 81000 \\
07 & $\mathrm{C}$ & $\mathrm{C}$ & $\mathrm{CH}_{3}$ & $\mathrm{CH} 3$ & ---- & 5900 \\
\hline
\end{tabular}<smiles>[H][Y19]1c2c(Br)cccc2c2nc3ccccc3c(N)c12</smiles>

\begin{tabular}{lllll}
\hline $\mathrm{ID}$ & $\mathrm{n}$ & $\mathrm{R}_{1}$ & $\mathrm{Y}$ & $\mathrm{IC}_{50}^{*}$ \\
\hline 08 & 1 & $\mathrm{H}$ & $\mathrm{CH}_{2}$ & 33000 \\
09 & 1 & $\mathrm{Cl}$ & $\mathrm{CH}_{2}$ & 10300 \\
10 & 2 & $\mathrm{H}$ & $\mathrm{CH}_{2}$ & 36000 \\
11 & 3 & $\mathrm{H}$ & $\mathrm{NH}$ & 6700 \\
\hline
\end{tabular}<smiles>[R1]Nc1c2c(cc3cc([R3])ccc13)-c1ccc([R])c([R])c1C2</smiles>

\begin{tabular}{cccccc}
\hline $\mathrm{ID}$ & $\mathrm{R}_{1}$ & $\mathrm{R}_{2}$ & $\mathrm{R}_{3}$ & $\mathrm{R}_{4}$ & $\mathrm{IC}_{50} *$ \\
\hline 12 & $\mathrm{H}$ & $\mathrm{H}$ & $\mathrm{H}$ & $\mathrm{H}$ & 3100 \\
13 & $\mathrm{H}$ & $\mathrm{H}$ & $\mathrm{F}$ & $\mathrm{H}$ & 5700 \\
14 & $\mathrm{H}$ & $\mathrm{OCH}_{3}$ & $\mathrm{H}$ & $\mathrm{H}$ & 3000 \\
15 & $\mathrm{H}$ & $\mathrm{H}$ & $\mathrm{H}$ & $\mathrm{CH}_{3}$ & 1600 \\
\hline
\end{tabular}<smiles>[R]C1([R])CC2CC1c1nc3ccccc3c(N)c12</smiles>

\begin{tabular}{lcccc}
\hline ID & $\mathrm{R}_{1}$ & $\mathrm{R}_{2}$ & $\mathrm{R}_{3}$ & $\mathrm{IC}_{50}{ }^{*}$ \\
\hline 16 & $\mathrm{CH}_{3}$ & $\mathrm{CH}_{3}$ & $\mathrm{CH}_{3}$ & 88000 \\
\hline
\end{tabular}

\begin{tabular}{|c|c|c|c|c|c|c|}
\hline \multicolumn{7}{|c|}{ Grupo Treino } \\
\hline ID & $\mathrm{IC}_{50} *$ & & & & & \\
\hline 17 & 26400 & & & & & \\
\hline ID & $\mathrm{R} 1$ & $\mathrm{R} 2$ & R3 & $\mathrm{R} 4$ & Estereoq. & $\mathrm{IC}_{50} *$ \\
\hline 18 & $\mathrm{CH}_{3}$ & $\mathrm{H}$ & $\mathrm{H}$ & $\mathrm{H}$ & 7R-11R & 316 \\
\hline 19 & $\mathrm{CH}_{3}$ & $\mathrm{H}$ & $\mathrm{H}$ & $\mathrm{H}$ & 7S-11S & 89 \\
\hline 20 & $\mathrm{CH}_{2} \mathrm{CH}_{3}$ & $\mathrm{H}$ & $\mathrm{H}$ & $\mathrm{H}$ & $7 \mathrm{~S}-11 \mathrm{R}$ & 79.3 \\
\hline 21 & $\mathrm{CH}_{3}$ & $\mathrm{H}$ & $\mathrm{H}$ & $\mathrm{CH}_{3}$ & ----- & 449 \\
\hline 22 & $\mathrm{CH}_{3}$ & $\mathrm{H}$ & $\mathrm{H}$ & $\mathrm{F}$ & 7R-11R & 2930 \\
\hline 23 & $\mathrm{CH}_{3}$ & $\mathrm{H}$ & $\mathrm{H}$ & $\mathrm{F}$ & 7S-11S & 138 \\
\hline 24 & $\mathrm{CH}_{3}$ & $\mathrm{~F}$ & $\mathrm{H}$ & $\mathrm{H}$ & ----- & 543 \\
\hline 25 & $\mathrm{CH}_{2} \mathrm{CH}_{3}$ & $\mathrm{H}$ & $\mathrm{H}$ & $\mathrm{CH}_{3}$ & 7R-11R & 115 \\
\hline 26 & $\mathrm{CH}_{2} \mathrm{CH}_{3}$ & $\mathrm{CH}_{3}$ & $\mathrm{H}$ & $\mathrm{H}$ & ----- & 512 \\
\hline 27 & $\mathrm{CH}_{3}$ & $\mathrm{H}$ & $\mathrm{H}$ & $\mathrm{Cl}$ & ----- & 236 \\
\hline 28 & $\mathrm{CH}_{2} \mathrm{CH}_{3}$ & $\mathrm{CH}_{3}$ & $\mathrm{H}$ & $\mathrm{CH}_{3}$ & ----- & 32.6 \\
\hline 29 & $\mathrm{CH}_{2} \mathrm{CH}_{3}$ & $\mathrm{H}$ & $\mathrm{H}$ & $\mathrm{F}$ & $7 \mathrm{~S}-11 \mathrm{~S}$ & 32.4 \\
\hline 30 & $\mathrm{CH}_{2} \mathrm{CH}_{3}$ & $\mathrm{H}$ & $\mathrm{H}$ & $\mathrm{F}$ & 7R-11R & 185 \\
\hline 31 & $\mathrm{CH}_{2} \mathrm{CH}_{3}$ & $\mathrm{~F}$ & $\mathrm{H}$ & $\mathrm{F}$ & ----- & 194 \\
\hline 32 & $\mathrm{CH}_{2} \mathrm{CH}_{3}$ & $\mathrm{Cl}$ & $\mathrm{H}$ & $\mathrm{H}$ & ----- & 331 \\
\hline 33 & $\mathrm{CH}_{2} \mathrm{CH}_{3}$ & $\mathrm{H}$ & $\mathrm{Cl}$ & $\mathrm{H}$ & ---- & 762 \\
\hline 34 & $\mathrm{CH}_{2} \mathrm{CH}_{3}$ & $\mathrm{H}$ & $\mathrm{H}$ & $\mathrm{Cl}$ & ----- & 15.8 \\
\hline 35 & $\mathrm{CH}_{2} \mathrm{CH}_{3}$ & $\mathrm{Cl}$ & $\mathrm{H}$ & $\mathrm{Cl}$ & ----- & 1210 \\
\hline 36 & $\mathrm{CH}_{3}$ & $\mathrm{H}$ & $\mathrm{H}$ & $\mathrm{H}$ & 148 & \\
\hline 37 & $\mathrm{CH}_{2} \mathrm{CH}_{3}$ & $\mathrm{H}$ & $\mathrm{H}$ & $\mathrm{H}$ & 63 & \\
\hline ID & $\mathrm{n}$ & $\mathrm{R}_{1}$ & $\mathrm{IC}_{50} *$ & & & \\
\hline 38 & 1 & $\mathrm{H}$ & 47,5 & & & \\
\hline 39 & 3 & $\mathrm{H}$ & 5,04 & & & \\
\hline 40 & 4 & $\mathrm{H}$ & 1,48 & & & \\
\hline 41 & 6 & $\mathrm{H}$ & 8,58 & & & \\
\hline 42 & 2 & $\mathrm{Cl}$ & 10,8 & & & \\
\hline
\end{tabular}


Tabela 1. continuação

ID

\begin{tabular}{ccccc}
\hline ID & $\mathrm{R}_{1}$ & $\mathrm{R}_{2}$ & $\mathrm{R}_{3}$ & $\mathrm{IC}_{50}{ }^{*}$ \\
\hline 50 & $\mathrm{H}$ & $\mathrm{H}$ & $\mathrm{H}$ & 41 \\
\hline
\end{tabular}<smiles>[R3]C1=CC2Cc3nc4cc([R3])c([R3])c([R2])c4c(N)c3C(C1)C2</smiles>

\begin{tabular}{ccccccc}
\hline ID & $\mathrm{R}_{1}$ & $\mathrm{R}_{2}$ & $\mathrm{R}_{3}$ & $\mathrm{R}_{4}$ & Estereoq. & $\mathrm{IC}_{50} *$ \\
\hline 51 & $\mathrm{CH}_{2} \mathrm{CH}_{3}$ & $\mathrm{H}$ & $\mathrm{H}$ & $\mathrm{CH} 3$ & $7 \mathrm{~S}-11 \mathrm{~S}$ & 347 \\
52 & $\mathrm{CH}_{3}$ & $\mathrm{~F}$ & $\mathrm{H}$ & $\mathrm{F}$ & ----- & 963 \\
53 & $\mathrm{CH}_{2} \mathrm{CH}_{3}$ & $\mathrm{~F}$ & $\mathrm{H}$ & $\mathrm{H}$ & ----- & 109 \\
\hline
\end{tabular}

* Nanomolar (nM) entre as quais se destaca a criação de modelos de interação inibidorenzima através da técnica de acoplamento molecular. Esse método busca identificar o encaixe dos inibidores, no sítio ativo da enzima, que apresenta maior complementaridade eletrônica e estérica em relação aos resíduos do sítio ativo. ${ }^{36} \mathrm{~A}$ fim de facilitar a análise visual dos resultados fornecidos pelo programa GOLD 3.11, as 3 soluções de maior pontuação foram reavaliadas com o auxílio do programa Drugscore, que avalia as distâncias interatômicas e os tipos de interação molecular com base naqueles encontrados em bancos de dados cristalográficos como CSD (Cambridge Structures Database) ou PDB (Protein Data Base). O resultado gráfico dessa análise, que pode ser realizado com auxílio do programa PYMOL, mostra se o perfil de interação de um dado ligante com o alvo terapêutico é comum no banco de dados ou não. Esse procedimento permitiu a identificação de soluções que mostram um padrão de interação semelhante ao observado para inibidores de AChE. Esses dados sugerem que as conformações selecionadas se aproximam da conformação bioativa desses compostos sendo, portanto, úteis para o desenvolvimento de modelos de QSAR 3D.

Alternativamente, utilizou-se o programa ROCS para sobrepor todos os confôrmeros de cada molécula sobre a molécula de huprina na sua conformação bioativa. Essa estratégia se baseia na hipótese de que os inibidores de BuChE devem adotar conformações similares quando no sítio ativo dessa enzima. Infelizmente não foi localizada nenhuma estrutura cristalográfica de $\mathrm{BuChE}$ em complexo com algum dos inibidores considerados nesse estudo. Por essa razão, utilizou-se como referência a conformação bioativa de huprina no sítio ativo de acetilcolinesterase. O programa ROCS calcula a melhor sobreposição entre as moléculas com base no volume estérico e no tipo de interação que elas realizam, como implementado na função ComboScore. A conformação com maior pontuação é aquela que melhor se sobrepõe sobre a molécula referência. As Figuras 2 e $1 \mathrm{~S}$ (material suplementar) mostram as conformações 3D obtidas através de cada uma dessas metodologias.

Após geração e análise das conformações, foram feitos o cálculo e a seleção dos descritores topográficos disponíveis no programa DRAGON 5.4. Inicialmente foram calculados 678 descritores tridimensionais (ex: WHIM, GETAWAY e Morse) que descrevem propriedades moleculares relacionadas ao formato, volume, propriedades eletrônicas etc. A fim de eliminar descritores que não contêm informações relevantes à atividade biológica e reduzir a colinearidade dos descritores entre si, adotou-se a seguinte estratégia; descritores com valor constante, baixa variância $(<0,01)$ ou com alta correlação entre si $(>0,97)$ foram excluídos. A seguir, utilizou-se o programa BUILDQSAR para

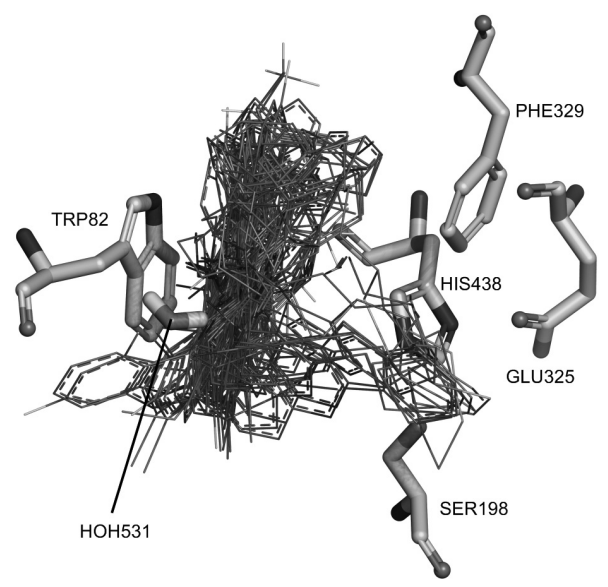

Figura 2. Padrão de interação dos inibidores de BuChE para CONF-GOLD (A) 
construir, através do método de busca sistemática, modelos de regressão linear múltipla (RLM) com até 4 descritores que apresentassem $r^{2}>0,78$. Nessa etapa todas as moléculas (conjunto treino e teste) foram utilizadas, a fim de não se introduzir algum tipo de tendência na etapa de seleção dos descritores. Dessa forma, essa metodologia visa identificar um subconjunto de descritores que estão de alguma forma relacionados com a atividade biológica e que podem ser úteis no desenvolvimento de modelos de QSAR robustos e com alto valor preditivo.

Os descritores presentes nos 10 melhores modelos de cada conjunto de conformações (Tabela 2) foram então agrupados, autoescalonados e utilizados nas análises de HCA, PCA e PLS.

No que se refere a CONF-GOLD, a análise do HCA - Hierarchical Cluster Analysis (Figura 2S) mostra que o conjunto de inibidores pode ser dividido em 4 agrupamentos principais (44\% de similaridade). Resultados qualitativamente similares foram obtidos para CONF-COMBO (dados não apresentados). Essa informação guiou a divisão do conjunto total de inibidores em grupo treino (144) e grupo teste (45-54) (Tabela 1), para fins de validação externa. Nessa divisão, compostos representantes de todos os agrupamentos foram aleatoriamente selecionados para compor o grupo teste. Essa estratégia garante que compostos estruturalmente diversos e com atividade biológica distribuída ao longo do eixo de potência estão presentes no grupo teste.

A análise de PCA (Figura 3) da CONF-GOLD demonstrou que dois componentes principais (PCs) representam 72,6\% da variância acumulada. A primeira componente explica a variação de potência entre os inibidores. Neste sentido, as moléculas representadas por círculos são muito potentes ( $\mathrm{pIC50}>$ 7,5); aquelas representadas por quadrados possuem potência mediana (pIC50 entre 6,0 e 7,49), enquanto as moléculas representadas por triângulos têm baixa potência (pIC50 < 5,99). A segunda componente por sua vez está relacionada com diferenças estruturais entre os inibidores. Assim, derivados de huprina estão representados por quadrados, ao passo que derivados de tacrina estão representados por círculos e triângulos.

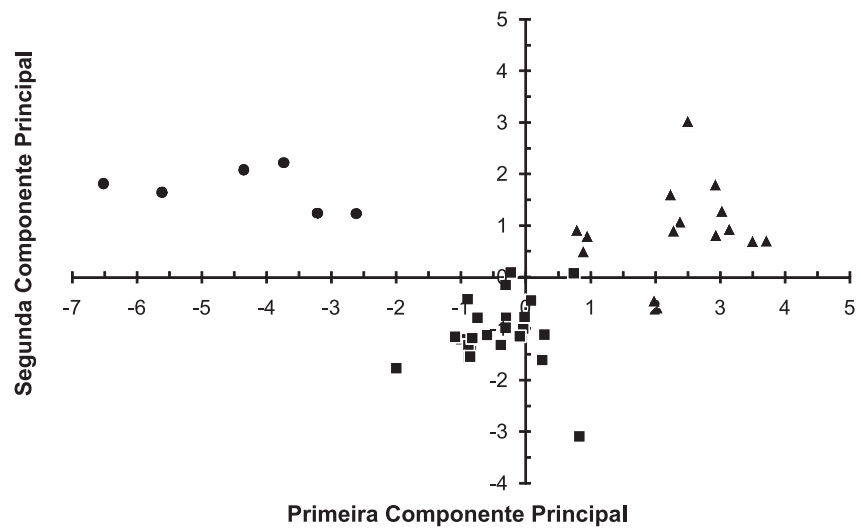

Figura 3. Análise de componentes principais de inibidores (grupo treino) de BuChE humana para CONF-GOLD

A análise da influência dos descritores sobre cada componente principal mostrou que os descritores Mor14v e Mor12u são as principais variáveis responsáveis pela diferenciação da potência, enquanto o descritor R5u é a variável mais importante para a distinção dos derivados de tacrina e huprina. Com relação à CONF-COMBO, 3 componentes contêm $71 \%$ da informação original, sendo que a primeira componente está relacionada com a potência dos compostos (Figura 3S). Esses resultados sugerem que os descritores selecionados capturam características estruturais importantes para atividade inibitória de BuChE e podem, portanto, ser utilizados no desenvolvimento de modelos de QSAR utilizando PLS.
Durante o desenvolvimento do modelo de PLS, a partir de CONFGOLD, descritores que apresentam baixas contribuições para o vetor de regressão foram eliminados. Dessa forma, o modelo final $\left(r^{2}=0,82\right.$ e $q^{2}=0,76$ com 2 PCs) apresenta apenas os 10 descritores em destaque na Tabela 2 (Figura 4).
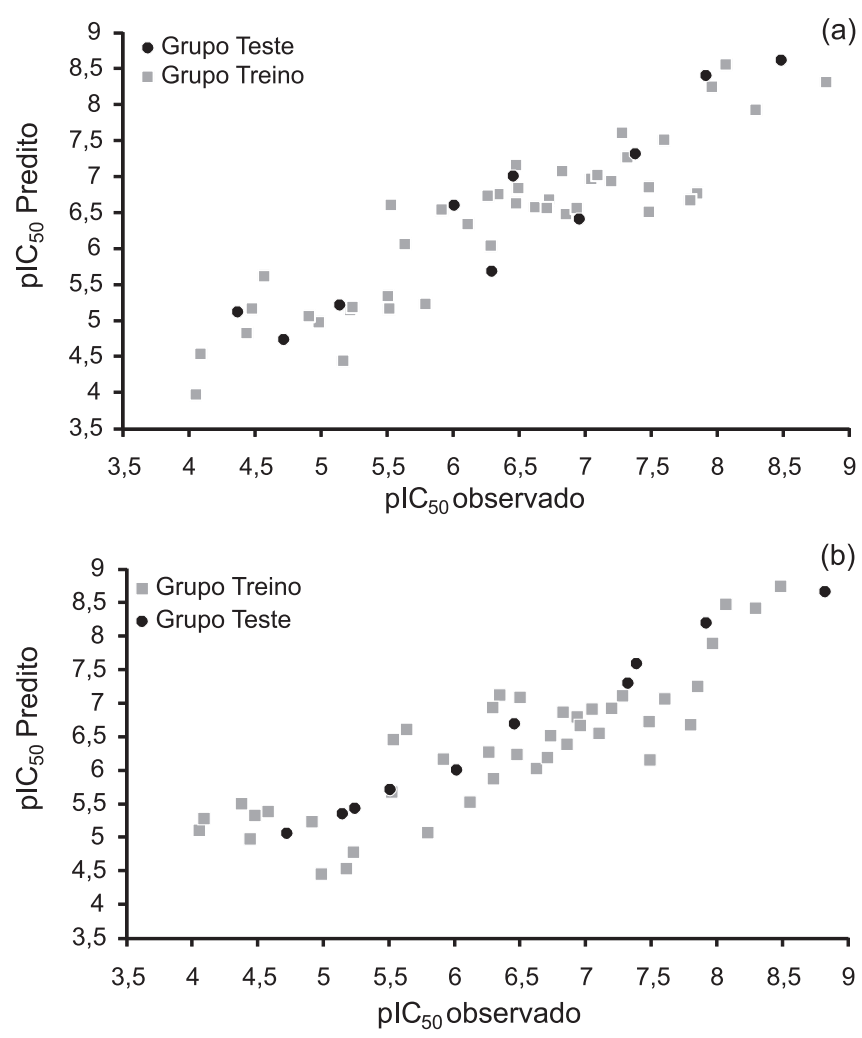

Figura 4. Gráfico de $\mathrm{pIC}_{50}$ predito x experimental para modelos de QSAR $3 D$ gerados a partir de CONF-GOLD (A) e CONF-COMBO (B)

Estratégia semelhante permitiu a identificação de 15 descritores que contribuem significantemente para o modelo de QSAR gerado a partir de CONF-COMBO $\left(r^{2}=0,79, q^{2}=0,70\right.$ com 2 PCs) (Figuras $4 \mathrm{~S}$ e 5). Vale a pena destacar que os descritores Mor12v e R2u são comuns aos dois modelos e outros 6 descritores presentes no modelo CONF-GOLD (Mor12u, Mor20m, Mor14v, Mor15v, HATs8u e R5u) são altamente correlacionados ( $>0,81)$ com descritores do modelo CONF-COMBO (Tabela 1S). Esse resultado sugere que os diferentes métodos de alinhamento molecular utilizados convergiram para modelos qualitativamente semelhantes. Ainda que os modelos apresentem ajustes internos razoáveis, estimar a capacidade preditiva deles a partir do valor de $q^{2}$ pode resultar em interpretações superestimadas (overfitting) de valor preditivo real. ${ }^{37}$ Por essa razão, utilizou-se um

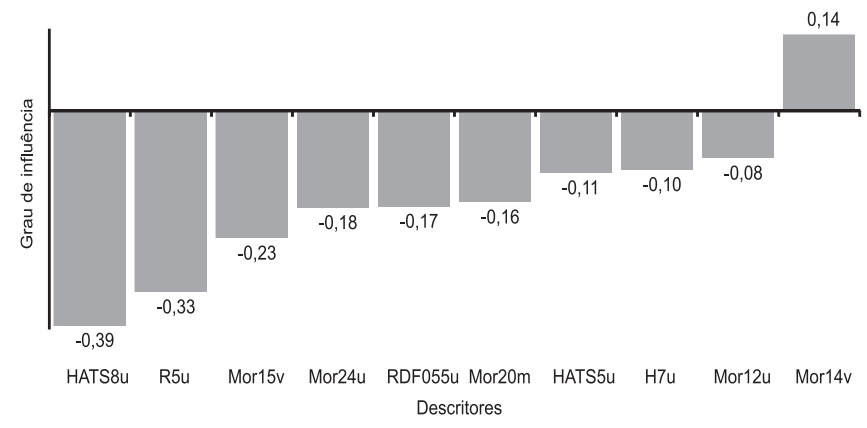

Figura 5. Gráfico do vetor de regressão do melhor modelo de QSAR para CONF-GOLD 
Tabela 2. Descritores selecionados através de Regressão Linear Múltipla

\begin{tabular}{|c|c|}
\hline Símbolo" & Descrição \\
\hline & CONFORMAÇÃO-GOLD \\
\hline G(N..O) & Soma das distancias geométricas entre N...O \\
\hline HATS5u & Influência da matriz de autocorrelação calculada entre átomos separados por 5 ligações químicas \\
\hline HATS8u & Influência da matriz de autocorrelação calculada entre átomos separados por 8 ligações químicas \\
\hline H6m & Matriz de autocorrelação H calculada entre átomos separados por 6 ligações químicas ajustada pela massa atômica \\
\hline H7u & Matriz de autocorrelação $H$ entre átomos separados por 7 ligações \\
\hline $\mathrm{L} 2 \mathrm{u}$ & $2^{\mathrm{a}}$ componente directional do índice WHIM \\
\hline Mor20m & Sinal 20 dos descritores 3D-MoRSE ajustado pela massa atômica) \\
\hline Mor31m & Sinal 31 dos descritores 3D-MoRSE ajustado pela massa atômica \\
\hline Mor12u & Sinal 12 dos descritores 3D-MoRSE \\
\hline Mor24u & Sinal 24 dos descritores 3D-MoRSE \\
\hline Mor08v & Sinal 08 dos descritores 3D-MoRSE ajustado pelo volume de Van Der Walls \\
\hline Mor12v & Sinal 12 dos descritores 3D-MoRSE ajustado pelo volume de Van Der Walls \\
\hline Mor14v & Sinal 14 dos descritores 3D-MoRSE ajustado pelo volume de Van Der Walls \\
\hline Mor15v & Sinal 15 dos descritores 3D-MoRSE ajustado pelo volume de Van Der Walls \\
\hline $\mathrm{R} 2 \mathrm{u}$ & Autocorrelação R calculada entre átomos separados por 2 ligações químicas \\
\hline R5u & Autocorrelação $\mathbf{R}$ calculada entre átomos separados por 5 ligações químicas \\
\hline \multirow[t]{2}{*}{$\underline{\text { RDF055u }}$} & Função de Distribuição Radial - 5,5 \\
\hline & CONFORMAÇÃO-COMBO \\
\hline$\overline{\mathrm{Dm}}$ & Índice de acessibilidade total ajustado pela massa atômica \\
\hline Ds & Índice de acessibilidade total D ajustado pelo estado eletro-topológico atômico \\
\hline Dv & Índice de acessibilidade total D ajustado pelo volume de Van Der Walls \\
\hline $\mathrm{G}(\mathrm{N} \ldots \mathrm{O})$ & Soma das distancias geométricas entre N...O \\
\hline $\mathrm{H} 0 \mathrm{v}$ & Índice de autocorrelação H do átomo, ajustado pelo volume de Van Der Walls \\
\hline HATS1m & Autocorrelação mediana entre átomos separados por uma ligação química, ajustada pela massa atômica \\
\hline HATS2m & Autocorrelação mediana entre átomos separados por 2 ligações químicas, ajustada pela massa atômica \\
\hline HATS5m & Autocorrelação mediana entre átomos separados por 5 ligações químicas, ajustada pela massa atômica \\
\hline HATS7m & Autocorrelação mediana entre átomos separados por 7 ligações químicas, ajustada pela massa atômica \\
\hline HATS2u & Autocorrelação mediana entre átomos separados por 2 ligações químicas \\
\hline HATS8u & Autocorrelação mediana entre átomos separados por 8 ligações químicas \\
\hline HOMA & Modelo de Oscilador Harmônico do índice de Aromaticidade \\
\hline ISH & Padronização das informações semelhantes ao leverage \\
\hline Mor12e & Sinal 12 dos descritores 3D-MoRSE ajustado pela eletronegatividade atômica de Sanderson \\
\hline Mor06u & Sinal 06 dos descritores 3D-MoRSE \\
\hline Mor12v & Sinal 12 dos descritores 3D-MoRSE ajustado pelo volume de Van Der Walls \\
\hline Mor20v & Sinal 20 dos descritores 3D-MoRSE ajustado pelo volume de Van Der Walls \\
\hline Mor29v & Sinal 29 dos descritores 3D-MoRSE ajustado pelo volume de Van Der Walls \\
\hline Mor30v & Sinal 30 dos descritores 3D-MoRSE ajustado pelo volume de Van Der Walls \\
\hline $\mathrm{R} 3 \mathrm{~m}$ & Autocorrelação R entre átomos separados por 3 ligações químicas ajustado pela massa atômica \\
\hline R5m & Autocorrelação $\mathbf{R}$ entre átomos separados por 5 ligações químicas ajustado pela massa atômica \\
\hline R6m & Autocorrelação R entre átomos separados por 6 ligações químicas ajustado pela massa atômica \\
\hline $\mathbf{R} 2 \mathbf{u}$ & Autocorrelação R entre átomos separados por 2 ligações químicas \\
\hline R5u & Autocorrelação R entre átomos separados por 5 ligações químicas \\
\hline $\mathbf{R 8 u +}$ & Autocorrelação R máxima entre átomos separados por 8 ligações químicas \\
\hline $\mathrm{R} 4 \mathrm{v}$ & Autocorrelação R entre átomos separados por 6 ligações químicas ajustado pelo volume de Van Der Walls) \\
\hline RDF035u & Função de Distribuição Radial - 3,5 \\
\hline RDF040m & Função de Distribuição Radial - 4,0, ajustada pela massa atômica \\
\hline
\end{tabular}

\# Descritores presentes nos modelo finais de QSAR estão destacados (negrito) 
conjunto de inibidores, não utilizados durante o desenvolvimento do modelo (grupo teste), para avaliar a capacidade preditiva real desses modelos.

Os resultados mostrados na Figura 4 e na Tabela 3 indicam que os modelos obtidos apresentam alto poder preditivo, uma vez que a diferença entre os valores de $\mathrm{pIC}_{50}$ experimental e $\mathrm{pIC}_{50}$ predito são inferiores a 0,75 unidades de $\log$ para CONF-GOLD $\left(r_{\text {pred }}^{2}=0,88\right) \mathrm{e}$ inferiores a 0,51 unidades de log para CONF-COMBO $\left(r_{\text {pred }}^{2}=0,90\right)$, exceto para o composto 45 (vide Tabela 3 ), cujo resíduo é de 1,12 unidade de $\log$.

Tabela 3. Valores de $\mathrm{pIC}_{50}$ predito para compostos do grupo teste

\begin{tabular}{lccccc}
\hline \multirow{2}{*}{ Moléculas } & & \multicolumn{2}{c}{ GOLD } & \multicolumn{2}{c}{ COMBO } \\
\cline { 3 - 6 } & pIC $_{50}$ & $\mathrm{pIC}_{50}$ & Resíduo & $\mathrm{pIC}_{50}$ & Resíduo \\
Predito & \\
\hline 44 & 4,72 & 4,74 & 0,02 & 4,99 & 0,27 \\
45 & 4,38 & 5,13 & 0,75 & 5,50 & 1,12 \\
46 & 5,15 & 5,22 & 0,07 & 5,36 & 0,21 \\
47 & 6,30 & 5,69 & $-0,61$ & 5,94 & $-0,36$ \\
48 & 7,92 & 8,41 & 0,49 & 8,27 & 0,35 \\
49 & 8,49 & 8,62 & 0,13 & 8,90 & 0,41 \\
50 & 7,39 & 7,32 & $-0,07$ & 7,40 & 0,01 \\
51 & 6,46 & 7,01 & 0,55 & 6,70 & 0,24 \\
52 & 6,02 & 6,61 & 0,59 & 5,99 & $-0,03$ \\
53 & 6,96 & 6,41 & $-0,55$ & 6,61 & $-0,35$ \\
\hline
\end{tabular}

Embora a capacidade de predizer a atividades de novos compostos seja um quesito essencial, ele não é suficiente para um bom modelo de QSAR. ${ }^{38}$ Adicionalmente, deve-se ser capaz de utilizar informações provenientes dos modelos de QSAR para auxiliar na elucidação de requisitos químicos e/ou estruturais importantes para atividade biológica. Isso pode ser feito através da interpretação dos descritores de maior relevância para o modelo de QSAR 3D, ou seja, aqueles que apresentam maiores coeficientes de regressão no modelo. A Figura 5 indica que são eles HATS8u, R5u e Mor14v para CONF-GOLD e HOMA e HATS8u para CONF-COMBO (Figura 4S).

HATS8u e R5u fazem parte dos descritores GETAWAY ( $G E$ ometry, Topology, and Atom-Weights AssemblY) que codificam informações relativas à conformação e podem descrever informações adicionais como massa atômica, polarizabilidade, volume de van der Waals e eletronegatividade. ${ }^{39,40}$ HATS8u é calculado a partir da matriz de influência molecular (Molecular influence matrix $\boldsymbol{H}$ ) ao passo que a variável R5u é calculada através da matriz influência/ distância (Influence/distance matrix $\boldsymbol{R}$ ) que combina a matriz de influência molecular com a distância geométrica interatômica da molécula. ${ }^{39}$ Moléculas compactas apresentam valores de R5u maiores (16: $\left.\mathrm{R} 5 \mathrm{u}=2,22, \mathrm{pIC}_{50}=4,06\right) \mathrm{e}$, por conseguinte, menor atividade inibitória sobre BuChE, ao passo que moléculas que podem adotar conformações mais estendidas exibem o comportamento contrário (40: $\left.\mathrm{R} 5 \mathrm{u}=1,33, \mathrm{pIC}_{50}=8,83\right)$. Isso pode estar associado à melhor ocupação do sítio ativo, impedindo assim o acesso do substrato ao sítio ativo da enzima.

Essa hipótese é corroborada pela análise de Mor14v, um descritor 3D-MoRSE (Molecular Representation of Structures based on Electron diffraction $)^{40-42}$ que descreve informações relativas ao volume ocupado pela molécula. ${ }^{43,44}$ Inibidores que ocupam a totalidade do sítio de ligação de BuChE como 40 (Mor14v=1,74, $\mathrm{pIC}_{50}=8,83$ ) tem valores de Mor $14 \mathrm{v}$ muito superior quando compa- rados à $\mathbf{0 3}\left(\mathrm{Mor} 14 \mathrm{v}=0,003, \mathrm{pIC}_{50}=7,85\right)$. O descritor HOMA, por sua vez mede o decréscimo da aromaticidade do sistema, ${ }^{45}$ ou seja, quanto menor for o valor do descritor, menor será a aromaticidade do composto; o valor máximo do descritor HOMA é 1. Resumindo, para vetor de regressão do modelo de QSAR 3D o descritor HOMA está diretamente relacionado com a atividade, portanto, quanto maior o valor de HOMA melhor a atividade biológica do composto. Geralmente, moléculas com HOMA alto e HATS8u baixo são moléculas potentes $\left(\mathbf{0 1}\right.$ : $\left.\mathrm{HOMA}=0,97, \mathrm{HATS} 8 \mathrm{u}=0,25, \mathrm{pIC}_{50}=7,28\right)$ enquanto moléculas que têm valor de HOMA e HATS8u altos (11: HOMA=0,60, HATS8u=0,82, $\left.\mathrm{pIC}_{50}=5,17\right)$ apresentam uma diminuição na potência. Os valores elevados de HATS8u são observados em moléculas altamente ramificadas que podem apresentar baixa complementaridade estérica no sítio ativo de BuChE. Dessa forma, a análise do vetor de regressão do modelo sugere que fatores estéricos são os principais responsáveis pela diferença de potência nessa série de inibidores de BuChE.

\section{CONCLUSÃO}

Modelos de QSAR 3D são amplamente empregados no planejamento de novos fármacos, contudo a qualidade e aplicabilidade desses modelos dependem diretamente da conformação utilizada no seu desenvolvimento. Nesse trabalho, conformações baseadas em informações provenientes do alvo macromolecular ou do ligante, na sua conformação bioativa, foram utilizadas. Em ambos os casos, modelos com qualidade estatística semelhantes e que destacam a importância de fatores estéricos para atividade biológica foram encontrados. De fato, os modelos de QSAR 3D apresentam capacidade preditiva comparável à do modelo de Holograma QSAR (2D) previamente descrito por Castilho e colaboradores $^{21}\left(r^{2}=0,928\right.$ e $\left.q^{2}=0,723\right)$, com a vantagem adicional de auxiliarem na elucidação dos requisitos físico-químicos e estruturais que são importantes para inibição de BuChE. Adicionalmente, os resultados desse trabalho sugerem que modelos de QSAR 3D desenvolvidos a partir de informações de uma única molécula na sua conformação bioativa podem auxiliar no estudo das relações entre a estrutura química e a atividade biológica de inibidores de BuChE. Esse fato, aliado ao bom poder preditivo do modelo, indica que os modelos de QSAR 3D descritos nesse trabalho poderão ser úteis no planejamento de inibidores mais potentes e seletivos de BuChE.

\section{MATERIAL SUPLEMENTAR}

Está disponível em http://quimicanova.sbq.org.br, na forma de arquivo .PDF, com acesso livre. Este material refere-se às Figuras $1 \mathrm{~S}$ a $4 \mathrm{~S}$ e à Tabela $1 \mathrm{~S}$ mencionadas no texto.

\section{REFERÊNCIAS}

1. Rzeszotarski, W. J. In Burger's Medicinal Chemistry And Drug Discovery; Abraham, D. A., ed.; WileyBlackwell: New York, 2003, cap. 13.

2. Mount, C.; Downton, C.; Nat. Med. 2006, 12, 780.

3. Forlenza, O. V.; Revista De Psiquiatria Clínica 2005, 3, 137.

4. Klafki, H-W.; Staufenbiel, M.; Kornhuber, J.; Wiltfang J.; Brain 2006, $129,2840$.

5. Lleó, A.; Greenberg, S. M.; Growdon, J. H.; Annu. Rev. Med. 2006, 57, 513.

6. Laferla, F. M.; Green, K. N.; Oddo, S.; Nat. Rev. Neurosci. 2007, 8, 499.

7. Rakonczay, Z.; Acta Biol Hung. 2003, 54, 183.

8. Vellas, B.; Froelich, L.; Sampaio, C.; Alzheimer's \& Dementia 2007, 3, 152. 
9. Johnson, C. N.; Roland, A.; Upton, N.; Drug Discov. Today Ther. Strat. 2004, 1,13 .

10. Amieva, H.; Phillips, L. H.; Sala, S. D.; Henry, J. D.; Brain 2004, 127, 949.

11. Selkoe, D. J.; Nature 1999, 399, A23.

12. Ghosh, A. K.; Kumaragurubaran, N.; Hong, L.; Kulkarni, S.; Xu, X.; Miller, H. B.; Reddy, D. S.; Weerasena, V.; Turner, R.; Chang, W.; Koelsch, G.; Tang, J.; Bioorg. Med. Chem. Lett. 2008, 18, 1031.

13. Hussain, I.; Hawkins, J.; Harrison, D.; Hille, C.; Wayne, G.; Cutler, L.; Buck, T.; Walter, D.; Demont, E.; Howes, C.; Naylor, A.; Jeffrey, P.; Gonzalez, M. I.; Dingwall, C.; Michel, A.; Redshaw, S.; Davis, J. B.; J. Neurochem. 2007, 100, 802.

14. Lane R. M.; Potkin, S. G.; Enz, A.; Int. J. Neuropsychopharmacol. 2005, 9,1 .

15. Darvesh, S.; Hopkins, D. A.; Geula, C.; Bioorg. Med. Chem. 2003, 5, 131.

16. Darvesh, S.; McDonald, R. S.; Darvesh, K. V.; Mataija, D.; Conrad, S.; Gomez, G.; Walsh, R.; Martin, E.; Bioorg Med Chem. 2007, 15, 6367.

17. Melnikova, I.; Nat. Rev. Drug Discovery 2007, 6, 341.

18. Giacobini, E.; Pharmacol. Res. 2004, 50, 433.

19. Greig, N. H.; Utsuki, T.; Ingram, D. K.; Wang, Y.; Pepeu, G.; Scali, C.; Yu, Q. S.; Mamczarz, J.; Holloway, H. W.; Giordano, T.; Chen, D.; Furukawa, K.; Sambamurti, K.; Brossi, A.; Lahiri, D. K.; Proc. Natl. Acad. Sci. U.S.A. 2005, 102, 17213.

20. Liston, D.R.; Nielsen, J. A.; Villalobos, A.; Chapin, D.; Jones, S. B.; Hubbard, S. T.; Shalaby, I. A.; Ramirez, A.; Nason, D.; White, W. F.; Eur. J. Pharmacol. 2004, 486, 9.

21. Castilho, M. S.; Guido, R. V. C.; Andricopulo, A. D.; Lett. Drug Des. Discovery 2007, 4, 106

22. Camps, P.; El-Achab, R.; Görbig, D. M.; Morral, J.; Muños-Torrero, D.; Badia, A.; Baños, J. E.; Vivas, N. M.; Barril, X.; Orozco, M.; Luque, F. J.; J. Med. Chem. 1999, 42, 3227.

23. Camps, P.; El-Achab, R.; Morral, J.; Muños-Torrero, D.; Badia, A.; Baños, J. E.; Vivas, N. M.; Barril, X.; Orozco, M.; Luque, F. J.; J. Med. Chem. 2000, 43, 4657.

24. Camps, P.; Gómez, E.; Muñoz-Torrero, D.; Badia, A.; Vivas, N. M.; Barril, X.; Orozco, M.; Luque, F. J.; J. Med. Chem. 2001, 44, 4733.
25. Camps, P.; Formosa, X.; Muñoz-Torrero, D.; Petrignet, J.; Badia, A.; Clos, M. V.; J. Med. Chem. 2005, 48, 1701.

26. Rampa, A.; Bisi, A.; Belluti, F.; Gobbi, S.; Valenti, P.; Andrisano, V.; Cavrini, V.; Cavalli, A.; Recantini, M.; Bioorg. Med. Chem. 2000, 8, 497.

27. Marco, J. L.; Los-Rios, C.; Carreiras, M. C.; Baños, J. E.; Badía, A.; Vivas, N. M.; Bioorg. Med. Chem. 2001, 9, 727.

28. Mckenna, M. T.; Proctor, G. R.; Young, L. C.; Harvey, A. L.; J. Med. Chem. 1997, 40, 3516.

29. Castilho, M. S.; Postigo, M. P.; de Paula, C. B.; Montanari, C. A.; Oliva, G.; Andricopulo, A. D.; Bioorg. Med. Chem. 2006, 14, 516.

30. Gohlke, H.; Hendlich, M.; Klebe, G.; J. Mol. Biol. 2000, $295,337$.

31. Gohlke, H.; Hendlich, M.; Klebe, G.; Perspe. Drug Discovery Des. 2000, 20, 115 .

32. Allen, F. H.; Acta Crystallogr. 2002, B58, 380.

33. Velec, H. F. G.; Gohlke, H.; Klebe, G.; J. Med. Chem. 2005, 48, 6296.

34. Nicholls, A.; MacCuish, N. E.; MacCuish, J. D.; J. Comput. Aided Mol. Des. 2004, 18, 451,

35. Lemmen C.; Lengauer T.; J. Comput. Aided Mol. Des. 2000, 14, 215.

36. Wermuth ,C. G.; Ganellin, C. R.; Lindberg, P.; Mitscher, L. A.; Pure Appl. Chem. 1998, 70, 1129.

37. Golbraikh A.; Tropsha A.; J. Mol. Graph. Model. 2002, 20, 269.

38. Castilho, M. S.; Guido, R. V.; Andricopulo, A. D.; Bioorg. Med. Chem. 2007, 15, 6242.

39. Consonni, V.; Todeschini, R.; Pavan, M.; J. Chem. Inform. Comput. Sci. 2002, 42, 682 .

40. Consonni, V.; Todeschini, R.; Pavan, M.; Gramatica, P.; J. Chem. Inform. Comput. Sci. 2002, 42, 693

41. Gasteiger, J.; Schuur, J. H.; Selzer, P.; J. Chem. Inform. Comput. Sci. 1996, 36,334

42. Gasteiger, J.; Schuur, J. H.; Anal. Chem. 1997, 69, 13, 2398.

43. Saíz-Urra, L.; González, M. P.; Teijeira, M.; Bioorg. Med. Chem. 2006, $14,7347$.

44. Gasteiger, J.; Sadowski, J.; Schuur, J.; Selzer, P.; Steinhauer, L.; Steinhauer, V.; J. Chem. Inform. Comput. Sci. 1996, 36, 1030.

45. Kruszewski, J.; Krygowski, T. M.; Tetrahedron Lett. 1972, 36, 3839. 


\section{ESTUDOS DE QSAR 3D PARA UM CONJUNTO DE INIBIDORES DE BUTIRILCOLINESTERASE HUMANA}

Humberto F. Freitas, Odailson S. Paz e Marcelo S. Castilho*

Departamento de Medicamentos, Faculdade de Farmácia, Universidade Federal da Bahia, Campus Universitário de Ondina, 40170-115 Salvador - BA, Brasil

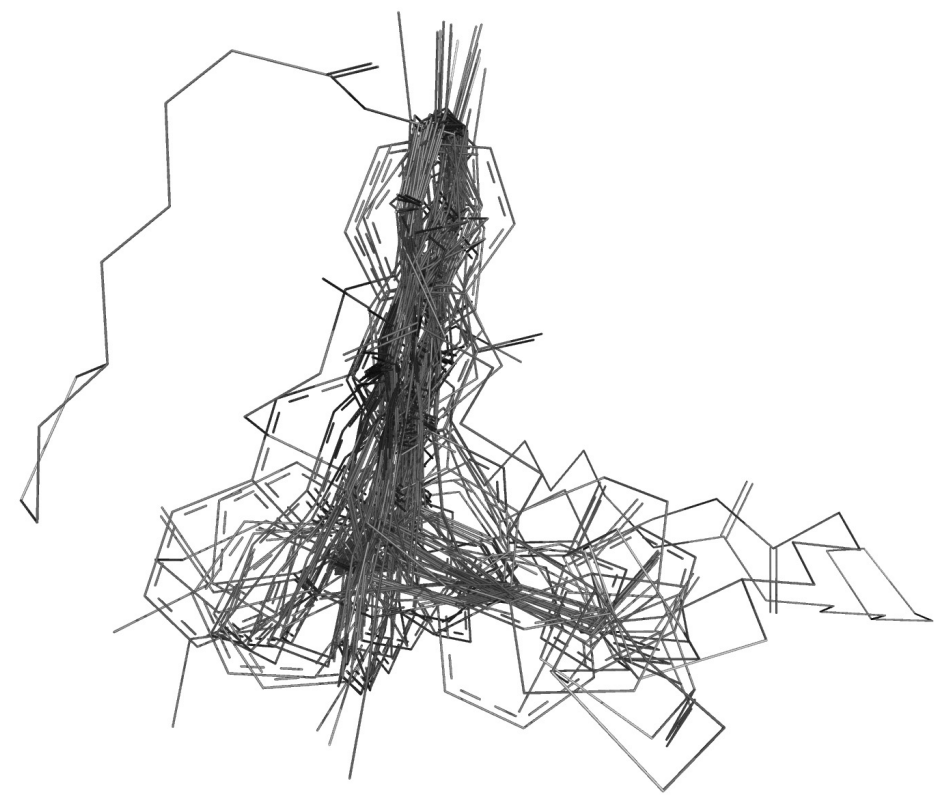

Figura 1S. Padrão de interação dos inibidores de BuChE para CONF-COMBO. Resíduos importantes para o mecanismo catalítico da enzima estão representados na Figura 2

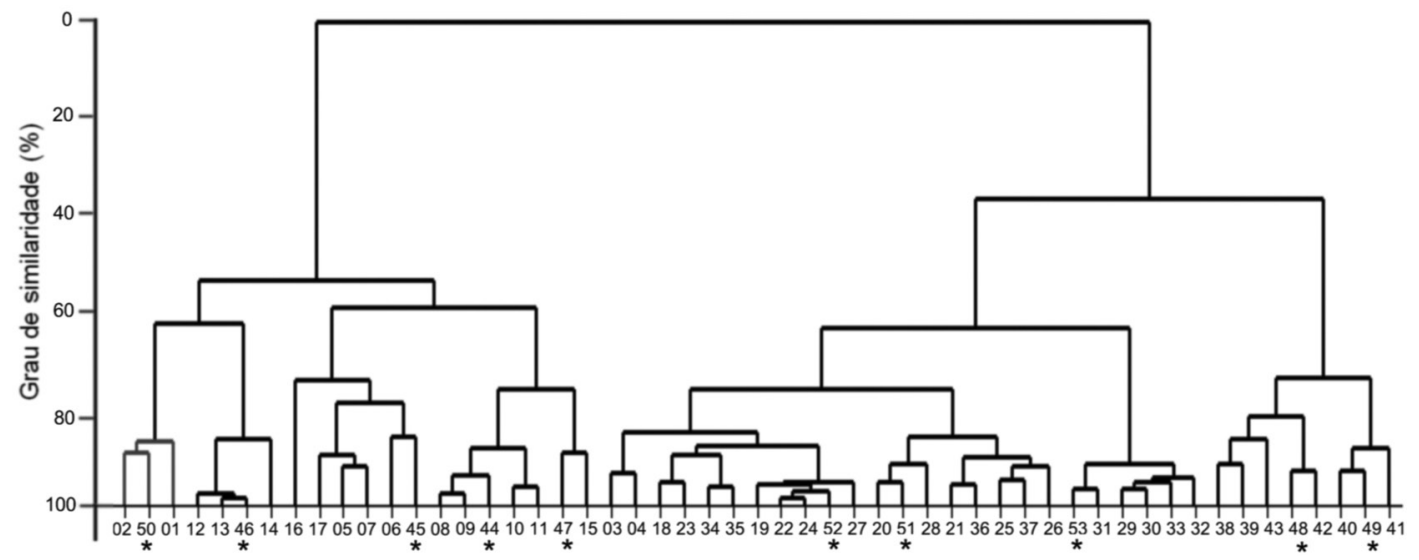

Figura 2S. Análise hierárquica de agrupamentos para inibidores de BuChE na CONF-GOLD. Inibidores que fazem parte do conjunto teste estão marcados com asterisco $(*)$

*e-mail: castilho@ufba.br 


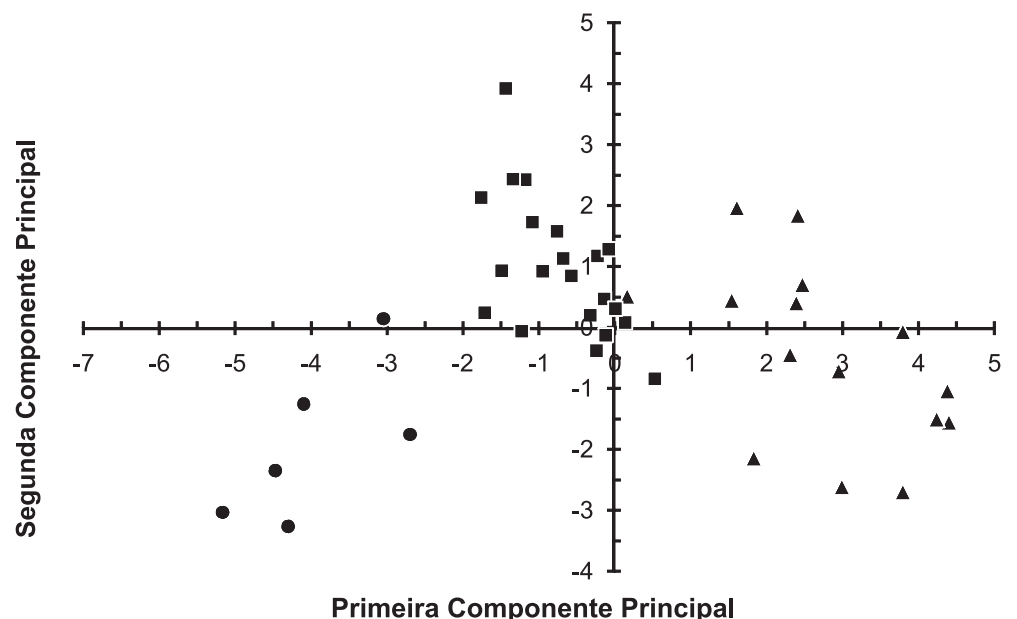

Figura 3S. Análise de componentes principais de inibidores (grupo treino) de BuChE humana para CONF-COMBO

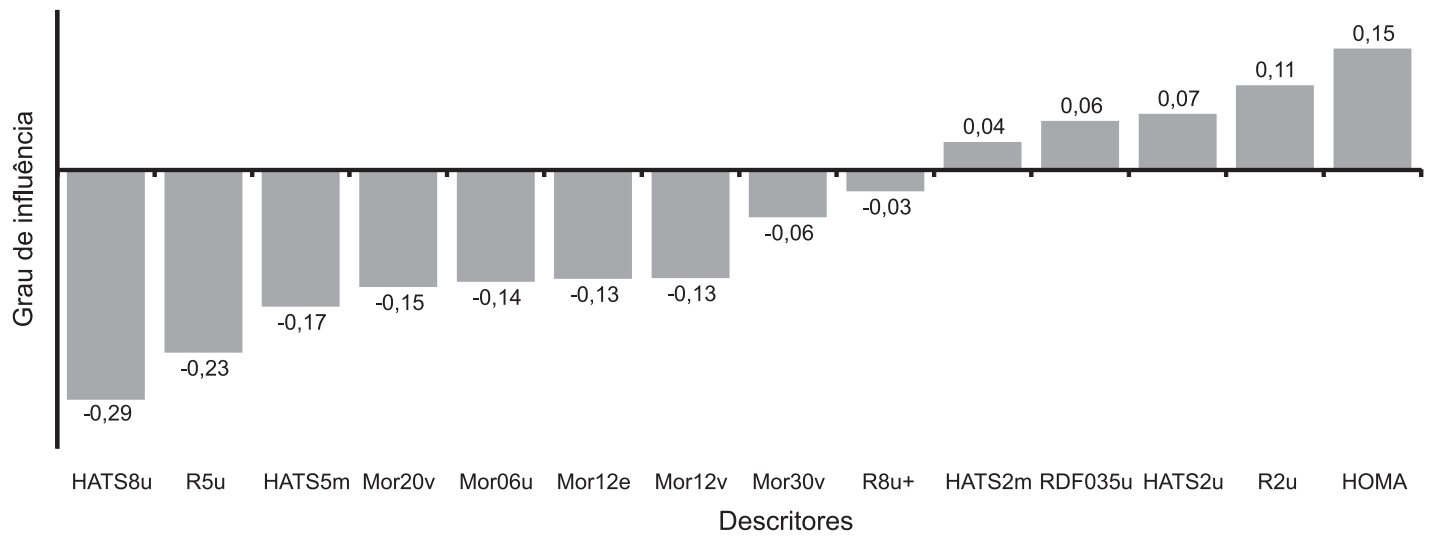

Figura 4S. Gráfico do vetor de regressão do melhor modelo de QSAR para CONF-COMBO

Tabela 1S. Grau de correlação entre os descritores dos modelos CONF-GOLD e CONF- COMBO

\begin{tabular}{|c|c|c|c|c|c|c|c|c|c|c|c|}
\hline & \multicolumn{10}{|c|}{ Descritores do modelo CONF-GOLD } \\
\hline & & RDF055u & Mor12u & Mor24u & Mor20m & Mor14v & Mor15v & H7u & HATS5u & HATS8u & R5u \\
\hline \multirow{15}{*}{$\begin{array}{l}0 \\
0 \\
\sum_{0}^{0} \\
0 \\
0 \\
\vdots \\
0 \\
0 \\
0 \\
0 \\
0 \\
0 \\
0 \\
0 \\
0 \\
0 \\
0 \\
0 \\
0 \\
0 \\
.0 \\
0 \\
0 \\
0 \\
0 \\
0\end{array}$} & HOMA & 0.068 & 0.178 & 0.117 & 0.329 & 0.335 & 0.331 & 0.002 & 0.106 & 0.226 & 0.036 \\
\hline & RDF035u & 0.150 & 0.239 & 0.241 & 0.470 & 0.532 & 0.562 & 0.003 & 0.118 & 0.012 & 0.283 \\
\hline & Mor06u & 0.067 & 0.003 & 0.001 & 0.040 & 0.009 & 0.005 & 0.056 & 0.024 & 0.027 & 0.032 \\
\hline & Mor12v & 0.533 & 0.838 & 0.235 & 0.673 & 0.835 & 0.710 & 0.478 & 0.495 & 0.188 & 0.005 \\
\hline & Mor20v & 0.308 & 0.484 & 0.217 & 0.914 & 0.764 & 0.829 & 0.107 & 0.177 & 0.195 & 0.138 \\
\hline & Mor30v & 0.482 & 0.567 & 0.089 & 0.350 & 0.495 & 0.343 & 0.519 & 0.357 & 0.197 & 0.017 \\
\hline & Mor12e & 0.525 & 0.907 & 0.180 & 0.447 & 0.651 & 0.498 & 0.554 & 0.604 & 0.138 & 0.018 \\
\hline & HATS2u & 0.610 & 0.711 & 0.040 & 0.232 & 0.526 & 0.323 & 0.492 & 0.598 & 0.049 & 0.001 \\
\hline & HATS8u & 0.189 & 0.291 & 0.048 & 0.221 & 0.288 & 0.258 & 0.242 & 0.115 & 0.952 & 0.029 \\
\hline & HATS2m & 0.136 & 0.196 & 0.029 & 0.048 & 0.242 & 0.158 & 0.076 & 0.187 & 0.151 & 0.002 \\
\hline & HATS5m & 0.048 & 0.154 & 0.088 & 0.035 & 0.014 & 0.009 & 0.263 & 0.051 & 0.047 & 0.136 \\
\hline & $\mathbf{R} 2 \mathbf{u}$ & 0.431 & 0.703 & 0.294 & 0.743 & 0.808 & 0.754 & 0.169 & 0.517 & 0.078 & 0.063 \\
\hline & R5u & 0.006 & 0.056 & 0.017 & 0.018 & 0.000 & 0.007 & 0.132 & 0.256 & 0.051 & 0.929 \\
\hline & R8u+ & 0.696 & 0.665 & 0.098 & 0.456 & 0.638 & 0.472 & 0.513 & 0.362 & 0.298 & 0.04 \\
\hline & R5m & 0.099 & 0.035 & 0.001 & 0.020 & 0.136 & 0.079 & 0.003 & 0.091 & 0.011 & 0.121 \\
\hline
\end{tabular}

